\title{
Open Texts on the Web, Taking the Next Step
}

\author{
Michael Doob
}

\section{Revolution to Evolution}

As noted by Steven E. Barkan [3] in his Notices piece, there is a revolution underway. Books both new and old are migrating from being published primarily in print to being published primarily in an electronic format. Many of the advantages of electronic publication are correctly identified in that article, and, indeed, this revolution allowing for open textbooks is certainly to be encouraged and applauded.

If freely available books are desired, they can be found easily online at, for example, the Open Textbook Library [6] or the American Institute of Mathematics [1]. There is a shortcoming, however. Almost all books appearing in electronic format are, well, just books. The format is essentially linear: start at the beginning and proceed forward to the end, just as with the scrolls found in the great library at Alexandria two thousand years ago. Although some typographical elements break this pattern, cross-references and indexes being examples, there is a basic expectation of linear structure in mathematical texts: Once a certain level of mathematical sophistication is presented at the beginning, the same level will continue through to the end; all the necessary elements for a proof are given before or immediately after its statement; similar or near-identical proofs are not repeated; forward references appear rarely if at

Michael Doob is professor of mathematics at The University of Manitoba. His email address is mdoob@ccu. umani toba.ca.

Members of the Editorial Board for Scripta Manent are: Jon Borwein, Thierry Bouche, John Ewing, Andrew Odlyzko, Ann Okerson.

DOI: http://dx.doi.org/10.1090/noti1141 all. As we write our new open texts, we have the opportunity to free ourselves from the chains of this restrictive structure. We should promote this renewal! In this note I'll give some examples of different approaches in this direction based on the wiki that I use for a first-year linear algebra course.

The mathematical world is fortunate to have LATEX as its lingua franca for typesetting mathematics. Although it was designed to produce traditional books, it has enough flexibility to adapt to more general formats. Most likely it will be our software of choice for the foreseeable future, and so its inclusion in any planning is important.

Almost all texts available in electronic form are in Portable Document Format (pdf). This format is a good first step, but most of these texts don't really exploit the full possibilities available.

\section{What pdf Has to Offer}

Putting books on the Web using a pdf file is easy, either by using pdfLTEX directly or by converting the standard LATEX output to a pdf file using intermediate software. While easy, we're still not using all the possibilities available.

Some pdf capabilities are really easy to use: first, the pdf file can be text-searchable, which will happen by default with most LTEX software. Next, the \1abe1-\ref, \cite-\bibentry and similar combinations can be live links. This is also easy to achieve: simply use the hyper ref package. This can also give appropriate links in a table of contents and an index (one might ask what the role of the index should be, given that it is easy to search for the first occurrence of any given term). It's surprising that there are any pdf files without these capabilities. 
In addition, color is available using the standard color package, and it can be used (judiciously, one hopes). Although it is easy, several of the available pdf files are simple conversions from existing texts that don't use these straightforward possibilities. This is the really low-hanging fruit; we should pick it.

What about graphics? Fortunately, accurate and high-quality graphics are available using standard programs such as TikZ[8] or Asymptote[2], and they may be used in conjunction with LATEX to create pdf files. There is a learning curve for either program that is a bit steep but nonetheless much easier than that for LATEX itself. The effort is worthwhile.

\section{Moving Beyond pdf}

While the pdf format has much to offer, there are disadvantages:

- The original design first appeared in 1993. It always has been superb at replicating printed text but not as successful with other media.

- Existing pdf files require specialized software for editing.

- Files with multimedia entities usually do not work with all viewers.

In contrast, the newer specifications for Web browser formats will allow both more flexibility and some newer features. Here are some examples:

- It is possible to use the less common but perhaps more useful method of folding text instead of adding links. In this case, clicking on a "link" opens (that is, inserts) new text at that point within the document. Clicking again makes the text disappear. A list of topics (the forest) may be given; clicking on any one of them adds more details (the trees); this process may be repeated for even more detail (the branches and then the leaves). The data is tree-structured rather than linear, and, as such, allows the same material to be viewed from different perspectives.

- Problem sets have always been included within textbooks. They, too, follow a linear format. The easier ones appear first followed by slightly harder ones. The use of an asterisk to indicate harder problems is an attempt to add information (depth) to this structure and goes back at least sixty years (see [5]). More recent books have made this mesh even finer. There now exists software (see [9]) that will parcel out problems from large problem banks, and, when appropriate, grade the answers. The problems given can be customized to the individual student: ones who are successful are given harder problems while those who are struggling get to repeat the effort until successful.
There is a wonderful example of an electronic textbook that moves beyond the pdf format. Rob Beezer's new online version of his linear algebra textbook [4] incorporates many of these advantages. It's worth a good look.

So we can see the evolution of electronic books taking place at this very moment. In fact they won't be much like books for long; we should call them open texts. The result of this evolution will be creative and exciting. It surely will be interesting to see if the next generation of students acquires its mathematical skills with greater ease and understanding than has heretofore been possible.

\section{References}

[1] American Institute of Mathematics Open Textbook Library website: http://aimath.org/textbooks.

[2] Asymptote website: http://sourceforge.net/ projects/asymptote/.

[3] STEVEN E. BARKAN, The open textbook revolution, Notices of the American Mathematical Society, 2012, 1447-1448.

[4] ROBERT BEEzer, A First Course in Linear Algebra, http://1inear.ups.edu/html/fcla.html

[5] G. BIRKHOFF and S. MAC LANE, A survey of modern algebra, The Macmillan Company, New York, 1953.

[6] A large number of freely available textbooks is available at the Open Textbook Library sponsored by the University of Minnesota at http://open . umn. edu/opentextbooks.

[7] Open Mathbook website: http://www. openmathbook.org/.

[8] TikZ website:/http://sourceforge.net/projects/ $\mathrm{pgf} /$.

[9] The Mathematical Association of America has a problem bank and associated software available at http://webwork.maa.org/.

\section{Some Graphic Examples}

A Matrix Row-Reduces Itself

One of the first algorithms taught in linear algebra is the one that takes a matrix and puts it into reduced row echelon form. There is a forest-tree problem: show the details of the algorithm but keep the overview of what's happening. Having an animation that runs at full speed or single-steps solves this problem. (See matrixanimation.gif, Notices online.)

$\left[\begin{array}{rrrrrrr}1 & 0 & 5 & -20 & -15 & -4 & -3 \\ 0 & 1 & -3 & 12 & 9 & 3 & 2 \\ 0 & 0 & 1 & -\frac{29}{8} & -\frac{21}{8} & -\frac{7}{8} & -\frac{1}{2} \\ 0 & 0 & -6 & 36 & 29 & 7 & 9 \\ 0 & 0 & -22 & 94 & 71 & 21 & 17\end{array}\right] \quad R_{3} \leftarrow \frac{1}{16} R_{3}$


American Mathematical Society

\section{AMS Grad Student Travel Grants}

Now providing support for doctoral student travel to the January Joint Mathematics Meetings or the AMS Sectional Meetings

- Listen to talks

- Meet active researchers

- Learn about professional issues and resources

- Make new professional connections

- Reconnect with colleagues

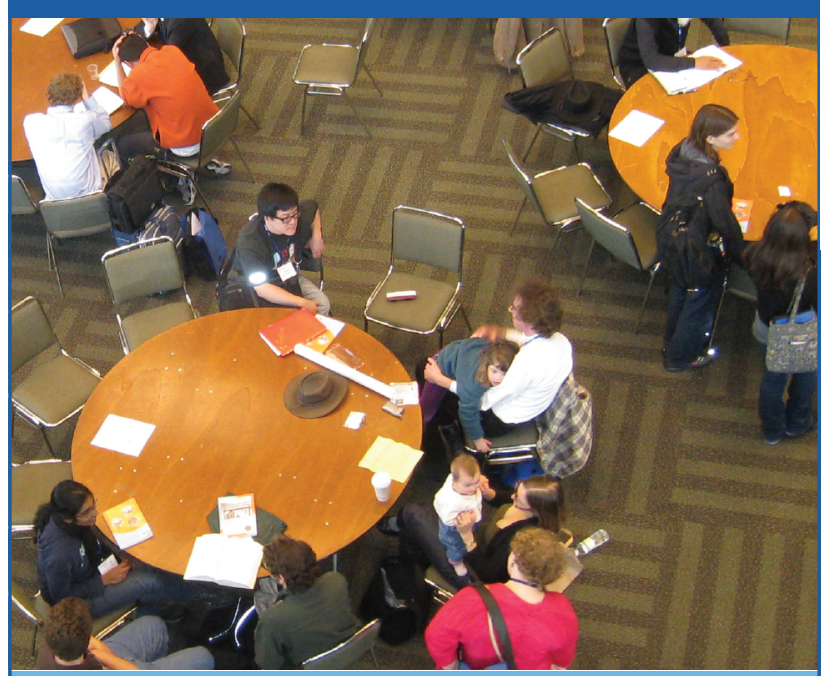

Check program announcements, eligibility requirements, and learn more at: www.ams.org/student-travel

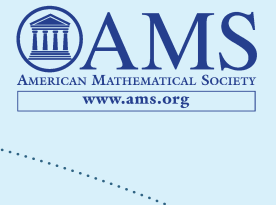

\section{A Cross Product Defines Itself}

The definition of the cross product is not very intuitive. I run the the following graphic telling the students that the red vector is the cross product of the blue vectors and ask for the properties. They get the orthogonality property immediately; with some prodding they get that the length is zero at 6:00 and 12:00 and maximum at 3:00 and 9:00. When I ask for a description of the length change as the hand rotates through $2 \pi$, someone always suggests $\sin (x)$. Using these properties, the definition of the cross product quickly emerges. (See crossproduct.gif, Notices online.)

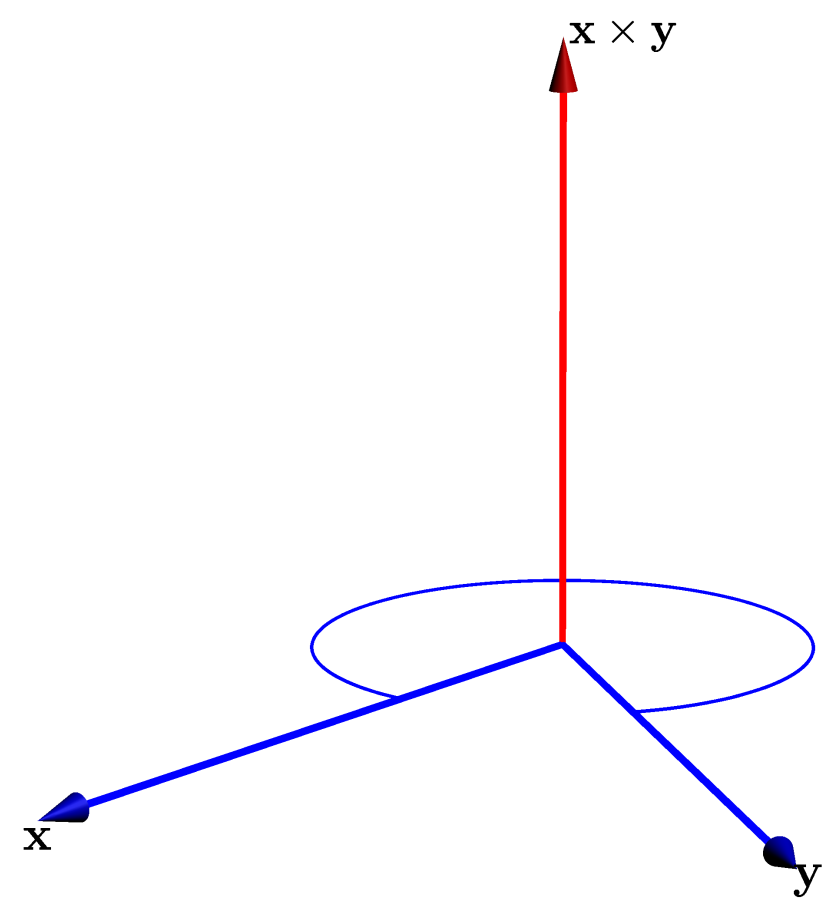

